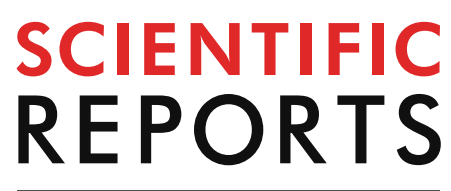

natureresearch

Check for updates

\title{
Stabilization of human
}

telomeric RNA G-quadruplex by the water-compatible optically pure and biologically-active metallohelices

\author{
Jaroslav Malina ${ }^{1}$, Peter Scott ${ }^{2}$ \& Viktor Brabec ${ }^{1 \bowtie}$ \\ RNA G-quadruplexes have been suggested to play key roles in fundamental biological processes and \\ are linked to human diseases. Thus, they also represent good potential therapeutic targets. Here, we \\ describe, using the methods of molecular biophysics, interactions of a series of biologically-active \\ supramolecular cationic metallohelices with human telomeric RNA G-quadruplex. We demonstrate \\ that the investigated metallohelices bind with a high affinity to human telomeric RNA G-quadruplex \\ and that their binding selectivity considerably differs depending on the dimensions and overall \\ shape of the metallohelices. Additionally, the investigated metallohelices inhibit DNA synthesis \\ on the RNA template containing four repeats of the human telomeric sequence by stabilizing the \\ RNA G-quadruplex structure. Collectively, the results of this study suggest that stabilization of RNA \\ sequences capable of G-quadruplex formation by metallohelices investigated in this work might \\ contribute to the mechanism of their biological activity.
}

Nucleic acids containing G-rich sequences can form a non-canonical tetrahelical structure called a G-quadruplex $^{1}$. In G-quadruplexes, four coplanar guanines are hydrogen-bonded to one another via Hoogsteen base pairs involving a total of eight hydrogen bonds, forming a planar complex (G-quartet). These G-quartets are stabilized by a central counterion, typically $\mathrm{K}^{+}$, and stack upon each other forming stable structures ${ }^{2}$.

Besides DNA, guanine-rich regions in RNA can also fold up into quadruplex structures. While DNA G-quadruplexes have been investigated for decades, such structures formed by RNA came into the focus of research considerably later. The main structural characteristics of RNA quadruplexes are similar to those of DNA G-quadruplexes, however there are significant differences. In contrast to DNA G-quadruplexes, which can adopt a number of quadruplex topologies ${ }^{3}$, RNA quadruplexes are believed to adopt only two structure types: a parallel G-quadruplex structure in which the strands all run in the same direction, and a more recently identified intramolecular antiparallel G-quadruplex structure ${ }^{4}$. Moreover, the stability of RNA G-quadruplexes is higher than that of DNA G-quadruplexes ${ }^{5}$.

Thousands of sequences capable of forming RNA G-quadruplexes have been identified within the human transcriptome $e^{6}$. There is circumstantial evidence that these structures exist in living cells ${ }^{7-10}$. They are frequently located in cellular regulatory sequences such as promoters, telomeres and the $5^{\prime}$ untranslated regions ( $5^{\prime}$ UTRs) of many genes, including genes of clinical interest ${ }^{11-13}$. Interestingly, $5^{\prime}$-UTR G-quadruplex structures have been shown to act as translational repressors ${ }^{6,13,14}$.

RNA G-quadruplexes have been suggested to modulate various biological mechanisms such as mRNA splicing, translation, localization, polyadenylation or regulation of miRNA precursor processing ${ }^{6,15,16}$. Additionally, RNA G-quadruplexes have been shown to be involved in the regulation of HIV-1 replication ${ }^{17}$ and G-rich sequences were found in the genom of several other viruses ${ }^{18-20}$.

Therefore, these structures represent good potential therapeutic targets ${ }^{21,22}$. Despite the growing knowledge about the importance of RNA quadruplexes, reports on their interaction with small molecules are relatively 


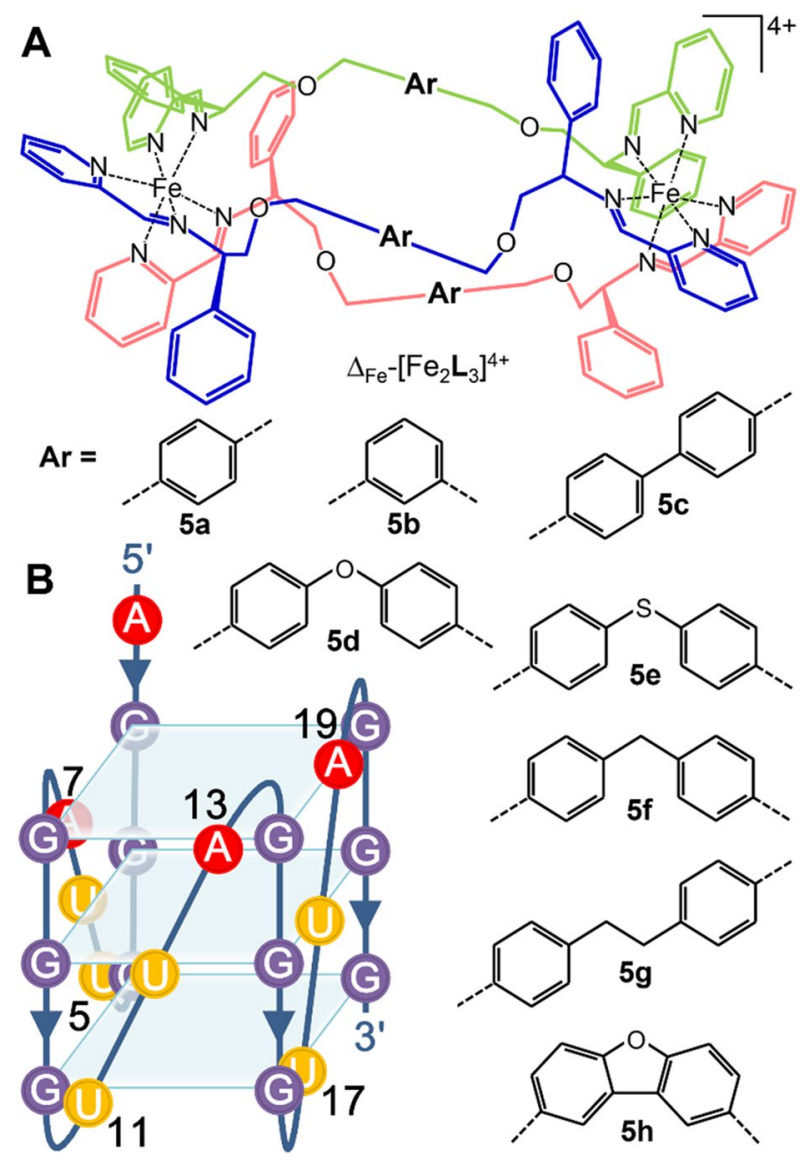

Figure 1. (A) Diastereomerically pure Fe(II) metallohelices 5a-h. (B) Scheme of the human telomeric RNA (TERRA) G-quadruplex.

scarce $^{23-25}$. For instance, the cationic 5, 10, 15, 20-tetra( $N$-methyl-4-pyridyl) porphyrin TMPyP4, which has been shown to stabilize many DNA G-quadruplexes ${ }^{26-28}$, can destabilize and unfold highly stable RNA G-quadruplexes $^{29,30}$. Additionally, naphthalenediimide derivatives have been shown to strongly stabilize a human telomeric DNA quadruplex, and a telomeric RNA quadruplex (TERRA), with weak stabilization of a duplex DNA ${ }^{31}$.

It has been shown that chiral $\mathrm{Fe}(\mathrm{II})$ and $\mathrm{Ni}(\mathrm{II})$ based supramolecular helicates $\left(\left[\mathrm{M}_{2}(\mathbf{I})_{3}\right] \mathrm{Cl}_{4} ; \mathbf{I}=\mathrm{C}_{25} \mathrm{H}_{20} \mathrm{~N}_{4}\right)$, as a type of metal complexes are able to stabilize human telomeric DNA G-quadruplex and inhibit telomerase activity $^{32,33}$. Another example of a chiral metallohelical complex 5a (see structure in Fig. 1A) capable of enantioselective stabilization of human telomeric hybrid DNA G-quadruplex and inhibition of telomerase activity was reported recently ${ }^{34}$. This compound developed by Scott and co-workers belongs to the class of cationic metallohelices that are based on helical arrays of fully-encapsulated Fe ions connected by different linking bridges (Fig. 1A $)^{35}$. Unlike above mentioned supramolecular helicates $\left[\mathrm{M}_{2}(\mathrm{I})_{3}\right] \mathrm{Cl}_{4}$, metallohelices are prepared as pure enantiomers and exhibit high kinetic stability in aqueous solutions ${ }^{35}$. Metallohelix 5a was shown to exhibit promising antimicrobial activity ${ }^{35}$ and served as a prototype compound for the development of a new class of metallohelices 5b-h (Fig. 1A) with structure-dependent activity against Gram-positive and -negative bacteria ${ }^{36}$. Additionally, we showed recently ${ }^{37}$ that for instance, metallohelices $\mathbf{5 b}$ and $\mathbf{5} \mathbf{f}$ accumulate in eukaryotic cells and that a significant fraction of the metallohelices accumulates in the cell nucleus allowing them to interact also with nuclear nucleic acids.

We recently studied the interaction of $\mathbf{5 b} \mathbf{b}-\mathbf{h}$ with various DNA G-quadruplexes including human telomeric G-quadruplex and our results revealed that some of the metallohelices prefer binding to G-quadruplexes over double-helical DNA (Ref. ${ }^{36}$ and unpublished data). The results suggest that the high binding affinity of $\mathbf{5 b}$ to DNA G-quadruplexes might contribute to its biological activity.

In this report, we demonstrate by using FRET melting assays, FID assays, gel electrophoresis and other methods that $\mathbf{5} \mathbf{a}-\mathbf{h}$ are able to recognize and stabilize human telomeric RNA (TERRA) G-quadruplex. Unlike its DNA counterpart, TERRA G-quadruplex folds in potassium ${ }^{21,38,39}$ and sodium ${ }^{40}$ solutions exclusively into a single topology - all strands parallel form (Fig. 1B).

\section{Results and discussion}

FID assays. The binding affinities of $\mathbf{5 a} \mathbf{a}-\mathbf{h}$ towards TERRA G-quadruplex were evaluated by using the fluorescent intercalator displacement (FID) assay. This widely used method to compare affinities of various ligands to different G-quadruplexes is based on the displacement of thiazole orange (TO ${ }^{41}$. The RNA G-quadruplex 


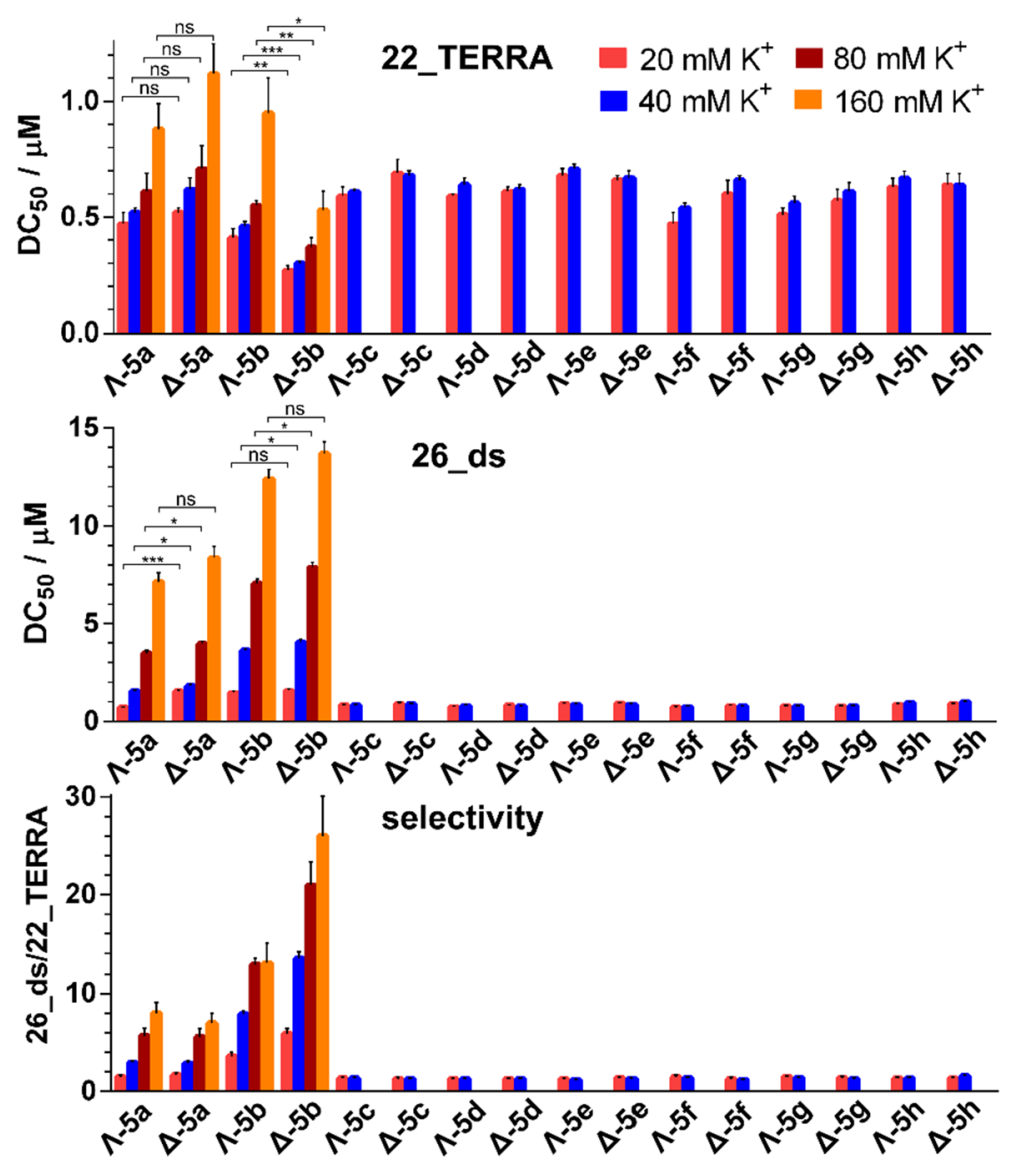

Figure 2. $\mathrm{DC}_{50}$ values $(\mu \mathrm{M})$ for the 22_TERRA (upper panel) and 26_ds (middle panel) determined by FID upon addition of the metallohelices in $10 \mathrm{mM}$ potassium phosphate buffer $(\mathrm{pH} 7)$ and various concentrations of $\mathrm{KCl}$. Binding selectivity (lower panel) was calculated as a ratio between $\mathrm{DC}_{50}$ values for the 26_ds and the 22_TERRA. Data shown are expressed as the mean of two independent experiments; error bars indicate the standard error of the mean. Symbols $\left({ }^{*},{ }^{* *},{ }^{* *}\right)$ and (ns) at the top of the bars indicate statistically significant difference $(\mathrm{p} \leq 0.1, \mathrm{p} \leq 0.05, \mathrm{p} \leq 0.01)$ and statistically insignificant difference $(\mathrm{p}>0.1)$, respectively.

(22_TERRA) and the duplex (26_ds) (for its sequence see "Experimental section") were treated with TO, yielding a fluorescence increase upon binding. The addition of metallohelices resulted in a decrease in the fluorescence intensity due to the displacement of bound TO (see Supplementary Figure S1), where the percent fluorescence reduction is directly related to the extent of binding. The concentrations of $\mathbf{5 a}-\mathbf{h}$ required to give a $50 \%$ decrease in $\mathrm{TO}$ fluorescence $\left(\mathrm{DC}_{50}\right.$ values) were determined and the results are shown in the bar graphs in Fig. 2 (for exact values see Supplementary Table S1). The $\mathrm{DC}_{50}$ values for $\mathbf{5 a}-\mathbf{h}$ obtained in the buffer containing $20 \mathrm{mM} \mathrm{K}^{+}$indicate that all metallohelices bound preferentially to 22_TERRA ( $\mathrm{DC}_{50}$ values ranging from 0.27 to $0.69 \mu \mathrm{M}$ ) versus 26 _ds duplex $\left(\mathrm{DC}_{50}\right.$ values ranging from 0.72 to $\left.1.58 \mu \mathrm{M}\right)$. The most potent G-quadruplex binder was $\boldsymbol{\Delta} \mathbf{- 5} \mathbf{b}$, followed by $\boldsymbol{\Lambda}-\mathbf{5 b}$ with the $\mathrm{DC}_{50}$ values of $0.27 \pm 0.02$ and $0.41 \pm 0.04 \mu \mathrm{M}$, respectively. It should be noted that ligands having $\mathrm{DC}_{50}$ values equal to or less than $0.5 \mu \mathrm{M}$ are regarded as excellent G-quadruplex binders $^{41}$. The selectivity of metallohelices towards the G-quadruplex motif was calculated as a ratio between the $\mathrm{DC}_{50}$ values obtained for the 26_ds and 22_TERRA. The highest selectivities of $3.6 \pm 0.41$ and $5.9 \pm 0.57$ were registered for $\Lambda \mathbf{- 5 b}$ and $\Delta \mathbf{- 5 b}$, respectively, while the selectivity values of remaining metallohelices were between 1.3 and 1.7 .

Electrostatic interactions play an important role in the binding of highly positively charged metallohelices to the negatively charged backbone of nucleic acids. In order to weaken the electrostatic attraction between the metallohelices and RNA, we performed the FID assays in the presence of increased ionic strength. Inspection of the data in Fig. 2 and Supplementary Table S2 reveals that the $\mathrm{DC}_{50}$ values recorded for the binding of $5 \mathbf{a}-\mathbf{h}$ to the 22_TERRA in $40 \mathrm{mM} \mathrm{K}{ }^{+}$were slightly increased and the same trend was observed for the 26_ds. The $\mathrm{DC}_{50}$ values of $\mathbf{5 a}$ and $\mathbf{5 b}$ for the $\mathbf{2 6}$ d ds were increased markedly more than those of $\mathbf{5} \mathbf{c}-\mathbf{h}$ and consequently, the binding selectivities of $\boldsymbol{\Lambda}-\mathbf{5 a}$ and $\boldsymbol{\Delta}$-5a towards 22_TERRA reached values of $3.0 \pm 0.2$ and $2.9 \pm 0.3$, respectively, while the binding selectivities of $\boldsymbol{\Lambda - 5} \mathbf{b}$ and $\Delta \mathbf{- 5} \mathbf{b}$ were increased even more to $7.9 \pm 0.4$ and $13.5 \pm 0.7$, respectively. The selectivities of $\mathbf{5} \mathbf{c}-\mathbf{h}$ remained almost unchanged in the range of $1.2-1.6$. The FID assays for $\mathbf{5 a}$ and $\mathbf{5 b}$ were repeated in the presence of 80 and $160 \mathrm{mM} \mathrm{K}^{+}$, which is close to the intracellular concentration of $\mathrm{K}^{+}(150 \mathrm{mM})$. Data in Fig. 2 (see also Supplementary Tables S3 and S4) show that the $\mathrm{DC}_{50}$ values kept on raising as a function of the concentration of $\mathrm{K}^{+}$and that the $\mathrm{DC}_{50}$ values for the 26_ds raised faster than those for the 22_TERRA. As 


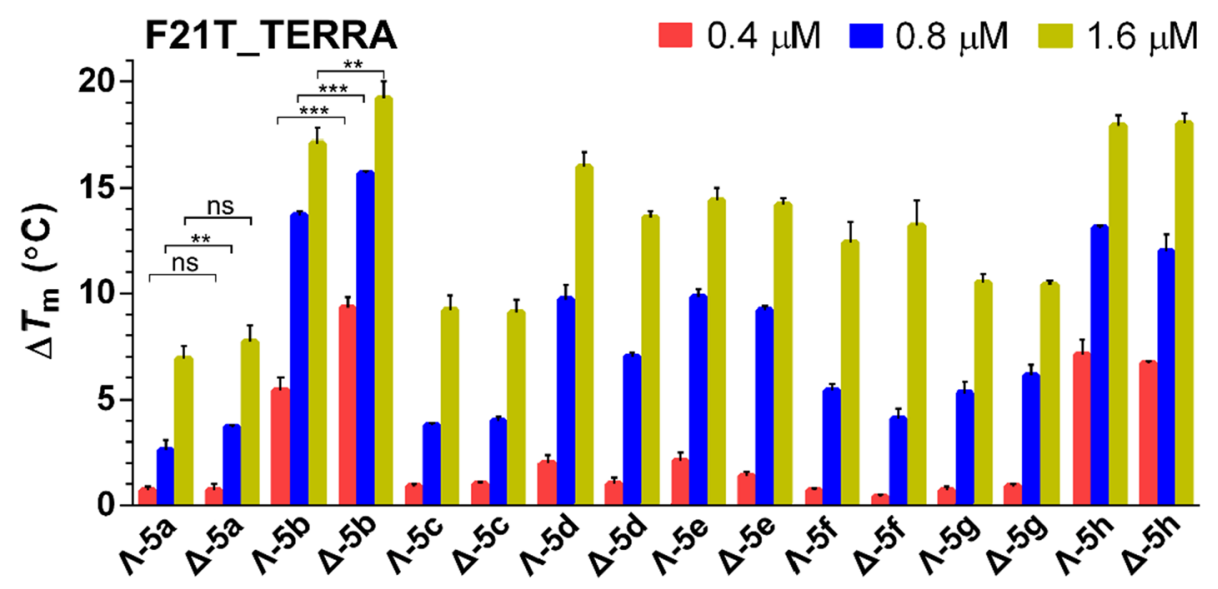

Figure 3. $\Delta T_{\mathrm{m}}$ values for the F21T_TERRA $(0.4 \mu \mathrm{M})$ determined by FRET upon addition of $0.4,0.8$, and $1.6 \mu \mathrm{M}$ metallohelices. The buffer conditions were $10 \mathrm{mM}$ potassium phosphate $(\mathrm{pH} 7)$ and $10 \mathrm{mM} \mathrm{KCl}$. Data shown are expressed as the mean of three independent experiments; error bars indicate the standard error of the mean. Symbols $\left({ }^{*},{ }^{* *},{ }^{* *}\right)$ and (ns) at the top of the bars indicate statistically significant difference ( $\mathrm{p} \leq 0.1$, $\mathrm{p} \leq 0.05, \mathrm{p} \leq 0.01)$ and statistically insignificant difference $(\mathrm{p}>0.1)$, respectively.

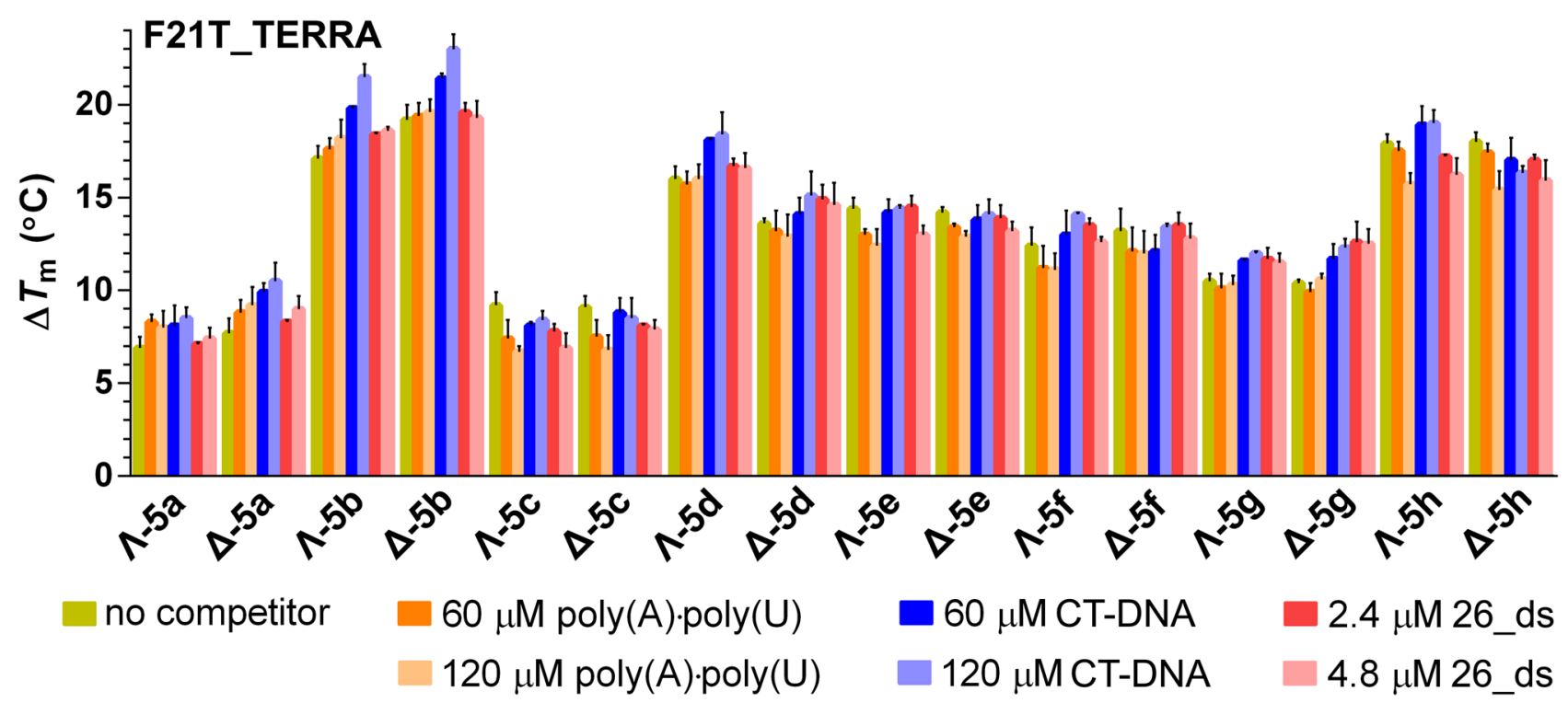

Figure 4. $\Delta T_{\mathrm{m}}$ values for the F21T_TERRA $(0.4 \mu \mathrm{M})$ determined by FRET upon addition of $1.6 \mu \mathrm{M}$ metallohelices in the presence of various concentrations of poly(A)·poly(U), CT-DNA and 26_ds. The buffer conditions were $10 \mathrm{mM}$ potassium phosphate $(\mathrm{pH} \mathrm{7)}$ and $10 \mathrm{mM} \mathrm{KCl}$. Data shown are expressed as the mean of three independent experiments; error bars indicate the standard error of the mean.

a result, the selectivities of $\mathbf{5 a}$ and $\mathbf{5 b}$ were gradually increasing, reaching maximum values of $8 \pm 1$ and $7 \pm 1$ for $\Lambda-5 \mathbf{a}$ and $\Delta-5 \mathbf{a}$ and $13 \pm 2$ and $26 \pm 4$ for $\Lambda-5 \mathbf{b}$ and $\Delta-\mathbf{5 b}$, respectively, in the presence of $160 \mathrm{mM} \mathrm{K}^{+}$.

FRET melting assays. To further investigate the interactions of $\mathbf{5 a - h}$ with the RNA G-quadruplex, we employed FRET melting assays. Examples of FRET melting curves obtained for the F21T_TERRA G-quadruplex and F26T_ds duplex upon the addition of the metallohelices are presented in Supplementary Figures S2 and S3, respectively. The $\Delta T_{\mathrm{m}}$ values for the F21T_TERRA summarized in the bar graph in Fig. 3 confirmed the good affinity of $\mathbf{5 a} \mathbf{a}$-h towards the G-quadruplex motif. It can be seen that the presence of $\mathbf{5 a}-\mathbf{h}$ at the concentration of $1.6 \mu \mathrm{M}$ increased the melting temperature of the F21T_TERRA by $7-19^{\circ} \mathrm{C}$ while the thermal stability of F26T_ds (Supplementary Figure S4) was just slightly increased by $\mathbf{5 b}$-h or remained unaffected by $\mathbf{5 a}$.

In the next step, we studied the ability of the metallohelices to stabilize the F21T_TERRA in the presence of increasing concentrations of competitors; the melting temperature was measured at $1.6 \mu \mathrm{M}$ concentration of $\mathbf{5 a}-\mathbf{h}$ in the presence of 60 and $120 \mu \mathrm{M}$ concentrations (per nucleotide) of synthetic double-stranded polynucleotide poly(A) poly(U), calf thymus (CT) DNA and 26_ds (examples of melting curves are shown in Supplementary Figure S5). The $\Delta T_{\mathrm{m}}$ values displayed in Fig. 4 show that the thermal stability of the F21T_TERRA in the presence of metallohelices was not markedly affected upon the addition of an excess of competitors. On the other hand, 

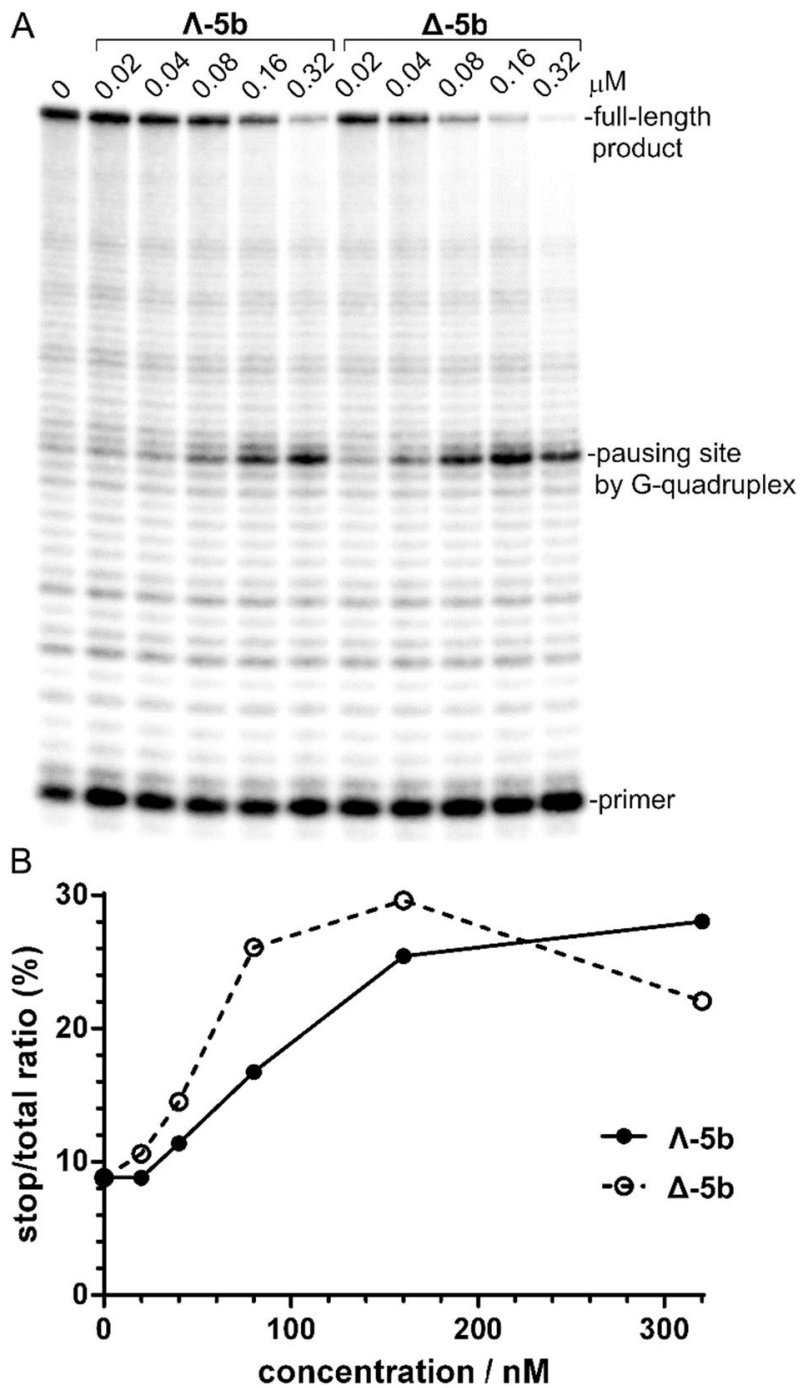

Figure 5. Inhibition of DNA synthesis catalyzed by ProtoScript II reverse transcriptase on the G-quadruplex containing HT4 RNA template $(40 \mathrm{nM})$ at $42^{\circ} \mathrm{C}$ in the presence of increasing concentrations $(20-320 \mathrm{nM})$ of $\Lambda-5 b$ and $\Delta-5 \mathbf{b}$. (A) Autoradiogram of $12 \%$ PAA sequencing gel. (B) The plot showing the ratio of the radiation corresponding to pausing sites to total radiation of the lane vs concentration of $\boldsymbol{\Lambda}-\mathbf{5 b}$ and $\Delta-\mathbf{5 b}$.

the low stabilizing effect of $\mathbf{5 b} \mathbf{b}-\mathbf{h}$ towards the F26T_ds was diminished to $0-1{ }^{\circ} \mathrm{C}$ upon addition of just $60 \mu \mathrm{M}$ $\operatorname{poly}(\mathrm{A}) \cdot \operatorname{poly}(\mathrm{U})$ (Supplementary Figure S4). Taken together, these results confirm the binding preference of the metallohelices for G-quadruplex RNA over double-stranded RNA and DNA.

The reviewer of this manuscript requested to mention in this manuscript that under certain circumstances, the FRET melting assay used to investigate the interactions of the investigated metallohelices with the RNA G-quadruplex may yield, false positive or false negative results. Therefore, we also performed control measurements of the $T_{\mathrm{m}}$ values using UV melting experiments (see Supplementary Figure S6). However, the UV melting experiments were difficult to evaluate accurately because RNA absorbs at $295 \mathrm{~nm}$ only slightly, whereas metallohelices yield in the UV region below $350 \mathrm{~nm}$ several narrow and intense peaks (shown for metallohelices $\mathbf{5 b}$ and $\mathbf{5 h}$ in Supplementary Figure S7). Thus, even a relatively small change of the intensity of the peaks and eventual their shifts when the temperature is changed, have a high impact on the shape of the melting curves recorded at $295 \mathrm{~nm}$, which makes their analysis more difficult. Additionally, the less accurate evaluation of the UV melting curves shown in Supplementary Figure S6 nevertheless confirms the conclusions drawn on the basis of the results of FRET melting experiments which were also confirmed by the results of the experiments in which the fluorescent intercalator displacement (FID) assay (Fig. 2) and Reverse transcriptase stop assay (Figs. 5 and 6) were used. It is also important to emphasize that FRET melting assay has been widely used to evaluate the stability of G-quadruplexes in the presence of various ligands, see Refs. ${ }^{42,43}$ as examples.

Reverse transcriptase stop assay. The following assay was employed to determine whether $\mathbf{5 a - h}$ interfere with the DNA synthesis catalyzed by reverse transcriptase (RT) on RNA template containing four repeats of the human telomeric sequence. It is a modification of a DNA polymerase arrest assay developed by Han et al. ${ }^{44}$ 


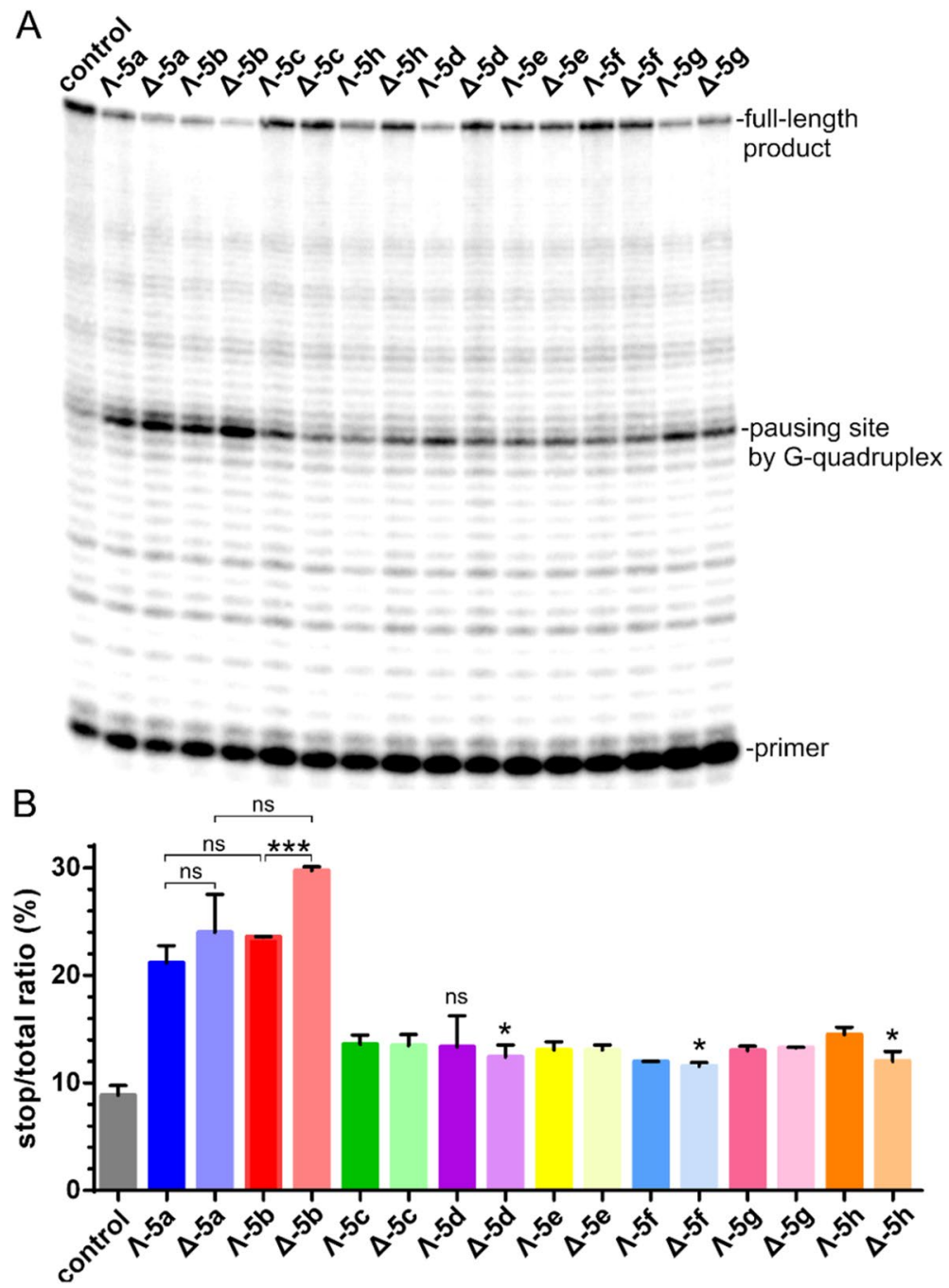

Figure 6. Inhibition of DNA synthesis catalyzed by ProtoScript II reverse transcriptase on the G-quadruplex containing HT4 RNA template $(40 \mathrm{nM})$ at $42{ }^{\circ} \mathrm{C}$ in the presence of $\mathbf{5 a}-\mathbf{h}(80 \mathrm{nM})$. (A) Autoradiogram of $12 \%$ PAA sequencing gel. (B) The bar graph showing the ratio of the radiation corresponding to pausing sites to total radiation of the lane. Data shown are expressed as the mean of two independent experiments; error bars indicate the standard error of the mean. Symbols $\left({ }^{*},{ }^{* *}\right)$ and (ns) at the top of the bars indicate statistically significant difference $(p \leq 0.1, p \leq 0.01)$ and statistically insignificant difference $(p>0.1)$, respectively. Data obtained for $\mathbf{5 a}-\mathbf{h}$ were tested against control and only values of $\mathrm{p}>0.05$ are indicated in the plot.

for DNA G-quadruplex-interactive ligands. We used ProtoScript II RT which is a recombinant M-MuLV RT with reduced RNase $\mathrm{H}$ activity and increased thermostability. The results of primer extension reactions catalyzed by ProtoScript II RT on the HT4 RNA template (for its sequence see "Experimental section") in the presence of increasing concentrations of $\boldsymbol{\Lambda}-\mathbf{5 b}$ and $\Delta-\mathbf{5 b}$ at $42^{\circ} \mathrm{C}$ are shown in Fig. 5.

As can be seen in the autoradiogram of the gel, the presence of a G-rich site in the RNA template led to slight pausing of the RT. The addition of $\mathbf{\Lambda - 5 b}$ and $\mathbf{\Delta - 5 b}$ caused an enhancement of pausing at the same site as that observed in the absence of metallohelices suggesting that both enantiomers increase pausing of the RT by stabilizing the G-quadruplex structure. The results were quantified and the plot in Fig. 5B reveals that both enantiomers are able to inhibit DNA polymerization at sub-micromolar concentrations and that $\Delta-\mathbf{5 b}$ is a slightly more efficient inhibitor than $\mathbf{\Lambda - 5} \mathbf{b}$, which is in agreement with the previous results.

In order to compare the potency of $\mathbf{5 a} \mathbf{a}-\mathbf{h}$ to inhibit the activity of RT, we performed the RT stop assay for all metallohelices at a fixed concentration of $80 \mathrm{nM}$ (Fig. 6). The results presented in the bar graph in Fig. 6B demonstrate that all metallohelices interfere to some extent with RT activity by stabilizing RNA G-quadruplex, but as expected $\boldsymbol{\Delta} \mathbf{- 5} \mathbf{b}$ was the most potent inhibitor followed by $\mathbf{\Lambda} \mathbf{- 5} \mathbf{b}$ and both enantiomers of $\mathbf{5 a}$.

RNase A digestion. In an effort to characterize the interaction of $\boldsymbol{\Lambda}-\mathbf{5 b}$ and $\mathbf{\Delta} \mathbf{- 5} \mathbf{b}$ with the 22_TERRA, we performed mapping experiment using RNase A. This endoribonuclease preferentially cuts single- over doublestranded RNA at the $3^{\prime}$-end of unpaired $\mathrm{C}$ and $\mathrm{U}$ residues making it a useful tool for investigating drug binding 
to the single-stranded regions of the RNA such as loops and bulges. The RNase A degradation pattern of $3^{\prime}-{ }^{32} \mathrm{P}$ end-labeled 22_TERRA is shown in Supplementary Figure S8, and as it can be observed (see also plots in Supplementary Figure S9), the cleavage at residues U17 and U11 was unchanged or only slightly enhanced at the doses of $\boldsymbol{\Lambda}-\mathbf{5 b}$ and $\boldsymbol{\Delta} \mathbf{- 5 b}$ corresponding to the metallohelix:G-quadruplex ratio 1:1-4:1. The presence of low concentrations of the metallohelices corresponding to the metallohelix:G-quadruplex ratio 1:1-2:1 had little effect on the degradation at residue U5, however when the metallohelix:G-quadruplex ratio exceeded 2:1 the reduction of RNase A cleavage was more pronounced (Supplementary Figure S9).

The low impact of $\mathbf{5 b}$ at 1:1 and 2:1 metallohelix:G-quadruplex ratios on the RNase A cleavage at residues U5, $\mathrm{U} 11$, and $\mathrm{U} 17$ located on the $3^{\prime}$-end of the G-quadruplex suggests that both enantiomers may bind by external stacking to the G-quartet on the opposite $5^{\prime}$-end of the G-quadruplex structure (see scheme in Fig. 1B).

2-aminopurine (2Ap) fluorescence studies. To further evaluate the interaction of $\mathbf{5 a} \mathbf{a}-\mathbf{h}$ with the human telomeric RNA G-quadruplex, we employed 22_TERRA labeled by 2-aminopurine (2Ap) at positions 7, 13, and 19 (see scheme in Fig. 1B). It was verified by CD spectroscopy (Supplementary Figure S10) that 2Ap modified 22_TERRA had the same conformation as the wild type. $2 \mathrm{Ap}$, the fluorescent analog of adenine, has been often used to probe the binding of small molecules to DNA and RNA ${ }^{45,46}$. The fluorescence intensity of $2 \mathrm{Ap}$ is efficiently quenched within the structure of double-stranded DNA and RNA, but it is enhanced when the base stacking or base pairing is perturbed. Other factors that affect 2 Ap fluorescence are collisions with other bases and biomolecular interactions ${ }^{47,48}$. The 2Ap-labeled 22_TERRA was first titrated with 5a and 5b in a series of experiments to select an appropriate metallohelix:RNA quadruplex ratio (Supplementary Figure S11). The plots show that both metallohelices efficiently reduced 2 Ap fluorescence and that $\mathbf{5 b}$ was slightly more efficient than $5 \mathrm{a}$. The intensity of $2 \mathrm{Ap}$ fluorescence was then measured at a fixed concentration of $2 \mathrm{Ap}$-labeled 22_TERRA $(2.5 \mu \mathrm{M})$ and two different concentrations $(2.5$ and $5 \mu \mathrm{M})$ of metallohelices corresponding to $1: 1$ and 2:1 metallohelix:G-quadruplex ratios. Results obtained for the 1:1 and 2:1 metallohelix:G-quadruplex ratios are displayed as bar graphs in Supplementary Figures S12 and S13, respectively. As it can be seen in both Figures, $\mathbf{5 a}-\mathbf{h}$ quenched the fluorescence of $2 \mathrm{Ap}$ regardless of its position in the RNA G-quadruplex. $\mathbf{\Lambda}-\mathbf{5 b}$ and $\mathbf{\Delta}-\mathbf{5 b}$ were the most potent quenchers of 2 Ap fluorescence, followed by the enantiomers of $\mathbf{5 a}$ and $\mathbf{5} \mathbf{d}$. The differences between $\Lambda$ - and $\Delta$-enantiomers were small, however, $\Delta-\mathbf{5}$ b was slightly more efficient quencher than $\Lambda-5 \mathbf{b}$. One can notice that $\mathbf{5 h}$ was markedly less active than other metallohelices in reducing the fluorescence of $2 \mathrm{Ap}$, but in this case, the results are influenced by the intrinsic fluorescence of $\mathbf{5 h}$ (see Supplementary Figure S14), which interferes with the fluorescence of $2 \mathrm{Ap}$ at $365 \mathrm{~nm}$.

The nearly equal reduction of the fluorescence of 2 Aps located in the loops at positions 7, 13, and 19 at both 1:1 and 2:1 metallohelix:G-quadruplex ratios suggests that $\mathbf{5 a - h}$ bind via external stacking to the terminal G-quartet on the $5^{\prime}$-side of the G-quadruplex as it has been proposed by RNase A footprinting. The binding of metallohelices might induce such conformational changes that enhance stacking interactions of 2 Aps with adjacent bases and increase the efficacy of the electron transfer quenching. It is also possible that the quenching action of the metallohelices originates from direct interactions of $\mathbf{5 a}-\mathbf{h}$ with $2 \mathrm{Aps}$.

One aspect of the binding mode of some ligands stabilizing DNA or RNA G quadruplexes is unfolding, or conformational changes of G-quadruplexes due to the binding of ligands and an efficient and widely used method to investigate these aspects of the binding mode is CD spectroscopy. The analysis of CD spectra of the mixture of 22_TERRA with metallohelices below $300 \mathrm{~nm}$ could not be interpreted in terms of the structural changes in the RNA G-quadruplex because the metallohelices also yield a very strong CD signal in the region of wavelengths in which RNA strongly absorbs (Supplementary Figure S15). Thus, interpretation of the CD spectra of the mixtures of the metallohelices and RNA in terms of conformational changes induced by metallohelices in RNA G quadruplexes is impossible.

\section{Conclusions}

In this study, we demonstrate that metallohelices $\mathbf{5 a}-\mathbf{h}$ bind with a high affinity to TERRA G-quadruplex and that their binding preferences for the G-quadruplex structure over double-stranded (ds) RNA differ considerably across the range of structures. Enantioselective recognition was observed for some of the metallohelices, particularly $\mathbf{5} \mathbf{a}$ and $\mathbf{5 b} \mathbf{b} \mathbf{\Delta} \mathbf{- 5} \mathbf{b}$ was shown to be the most potent G-quadruplex binder followed by $\boldsymbol{\Lambda}-\mathbf{5 b}$ and by the enantiomers of $\mathbf{5} \mathbf{a}$. Interestingly, both enantiomers of $\mathbf{5} \mathbf{b}$ exhibited also the highest affinity towards various DNA G-quadruplexes including one formed from the human telomeric sequence. Metallohelices $\mathbf{5} \mathbf{c}-\mathbf{h}$ also preferred binding to TERRA G-quadruplex over dsRNA but their binding selectivity was much lower, as compared to $\mathbf{5 b}$ and 5 a.

The higher binding affinity of $\mathbf{5 a}$ and its meta analog $\mathbf{5 b}$ to TERRA G-quadruplex might be associated with different dimensions and overall shape of these two metallohelices compared to $\mathbf{5 c}-\mathbf{h}$. $\mathbf{5 b}$ has the shortest intermetallic distance of $12.4 \AA$, followed by $\mathbf{5 a}$ and $\mathbf{5 h}$ with $14 \AA$ and $14.4 \AA$, respectively. The intermetallic distances in $\mathbf{5} \mathbf{c}-\mathbf{g}$ are markedly longer $>17 \AA$. The shorter Fe-Fe distance provides $\mathbf{5} \mathbf{a}$ and $\mathbf{5 b}$ with higher charge density and gives these compounds a more compact shape which seems to be more favorable for the binding to G-quadruplexes and less favorable for the binding to double-helical RNA. The longer metallohelices $\mathbf{5 c}-\mathbf{h}$ exhibit higher affinity towards double-helical RNA than $\mathbf{5 a}$ and $\mathbf{5 b}$ which leads to their lower binding selectivities against G-quadruplexes.

The binding selectivity of $\mathbf{5 a}$ and particularly $\mathbf{5 b}$ to the TERRA G-quadruplex increases with increasing ionic strength. This is because the presence of cations weakened the binding of $\mathbf{5 a}$ and $\mathbf{5} \mathbf{b}$ to dsRNA more than to the G-quadruplex structure. It suggests that the electrostatic forces are mainly involved in the interaction of $\mathbf{5 a}$ and 5b with dsRNA and play a less important role in binding to the G-quadruplex. On the contrary, the binding affinities of longer metallohelices $\mathbf{5 c}-\mathbf{h}$ to the G-quadruplex and dsRNA were affected to a similar degree by the 
presence of a higher concentration of $\mathrm{K}^{+}$which resulted in a negligible effect of the ionic strength on the binding selectivities. It seems that the binding of $\mathbf{5} \mathbf{c}-\mathbf{h}$ to dsRNA is not predominantly driven by the electrostatic forces or that the strengthening of another kind of interaction between the metallohelix and the double-helix compensates for the suppression of the electrostatic attraction in high salt concentration.

The presence of just $80 \mathrm{nM}$ concentration of $\mathbf{5} \mathbf{a}-\mathbf{h}$ was shown to inhibit DNA synthesis on the RNA template containing four repeats of the human telomeric sequence by stabilizing the G-quadruplex structure. The highest inhibitory effect was observed for $\Delta-\mathbf{5 b}$, followed by $\Lambda-\mathbf{5 b}$ and both enantiomers of $\mathbf{5 a}$.

The binding mode of the $\mathbf{5} \mathbf{a}-\mathbf{h}$ was not fully resolved, however, the results from the experiments with $2 \mathrm{Ap}$ labeled G-quadruplex and the cleavage by RNase A suggest that the metallohelices stack externally to the terminal G-quartet on the $5^{\prime}$-side of the TERRA G-quadruplex. Stacking interaction with the terminal G-quartet has been previously proposed for the binding of 5a with human telomeric DNA G-quadruple ${ }^{34}$. In summary, our results show that, 5a-h metallohelices are potent stabilizers of TERRA G-quadruplex and act as inhibitors of reverse transcription on the template containing four repeats of human telomeric sequence. If we take into account that metallohelices are able to accumulate inside cells and in the nucleus, we can speculate that stabilization of RNA sequences capable of G-quadruplex formation by metallohelices might contribute to the mechanism of their biological activity.

\section{Experimental section}

Chemicals and reagents. The metallohelices $\mathbf{5 a}-\mathbf{5 h}$ were synthesized and characterized, as described previously $^{36}$. The synthetic oligoribonucleotides and oligodeoxyribonucleotides used in this work were purchased from Eurofins Genomics (Ebersberg, Germany). T4 polynucleotide kinase and ProtoScript II reverse transcriptase were purchased from New England Biolabs (Beverly, MA). Calf thymus DNA (CT-DNA), poly(A)·poly(U), thiazole orange (TO) and RNase A were from Sigma-Aldrich (Prague, Czech Republic). $\left[\gamma^{-32} \mathrm{P}\right]$-ATP was from Hartmann analytic GmbH (Braunschweig, Germany). Acrylamide and bis(acrylamide) were from Merck KgaA (Darmstadt, Germany).

FID measurements. Oligoribonucleotides 22_TERRA, 5'-AGGGUUAGGGUUAGGGUUAGGG-3' and 26_ds hairpin 5'-CAAUCGGAUCGAAUUCGAUCCGAUUG-3' $(6.25 \mu \mathrm{M})$ were annealed in $10 \mathrm{mM}$ potassium phosphate buffer ( $\mathrm{pH} 7$ ) and in the presence of various concentrations of $\mathrm{KCl}$ by heating to $85^{\circ} \mathrm{C}$ for $3 \mathrm{~min}$ followed by slow cooling to room temperature. Thiazole orange solutions $(10 \mathrm{mM})$ in DMSO were prepared fresh each week. Oligoribonucleotides $(0.25 \mu \mathrm{M})$ were mixed with $0.5 \mu \mathrm{M}$ thiazole orange in a $1 \mathrm{~cm}$ quartz cuvette in a total volume of $2.5 \mathrm{~mL}$ and titrated with metallohelices. Small aliquots (typically $2.5 \mu \mathrm{L}$ ) of concentrated solutions (typically $1 \times 10^{-4} \mathrm{M}$ ) of metallohelices were added to the mixture. Samples were vigorously mixed by pipetting and left to equilibrate for $3 \mathrm{~min}$ at room temperature before data taking. Measurements were performed with the Varian Cary Eclipse spectrofluorophotometer using the following parameters: the excitation and emission wavelengths were set to $501 \mathrm{~nm}$ and $538 \mathrm{~nm}$, respectively, widths of the excitation and emission slit were $10 \mathrm{~nm}$, and the averaging time was set to $3 \mathrm{~s}$.

FRET melting assays. The solutions of the fluorescent-labeled (donor fluorophore FAM, 6-carboxyfluorescein; acceptor fluorophore TAMRA, 6-carboxytetramethylrhodamine) oligoribonucleotides F21T_TERRA, 5'-FAM-GGGUUAGGGUUAGGGUUAGGG-TAMRA-3', and F26T_ds hairpin, 5'-FAM-CAAUCGGAUCGA AUUCGAUCCGAUUG-TAMRA-3' $(4 \mu \mathrm{M})$ were annealed in $10 \mathrm{mM}$ potassium phosphate buffer $(\mathrm{pH} 7)$ and $10 \mathrm{mM} \mathrm{KCl}$ by heating to $85^{\circ} \mathrm{C}$ for $3 \mathrm{~min}$ followed by slow cooling to room temperature. Oligoribonucleotides $(0.4 \mu \mathrm{M})$ were either mixed with $0.4,0.8$, and $1.6 \mu \mathrm{M}$ metallohelices in the absence of the competitors or were added at the concentration of $1.6 \mu \mathrm{M}$ in the presence of various concentrations of poly(A).poly(U), CT-DNA or 26_ds hairpin. Samples were prepared in a total volume of $40 \mu \mathrm{L}$ in $0.2 \mathrm{~mL}$ microtubes. Measurements were carried out using the real-time PCR instrument RotorGene 6000 (Corbett Research). The excitation and detection wavelengths were set to $470 \pm 10 \mathrm{~nm}$ and $510 \pm 5 \mathrm{~nm}$, respectively, and the temperature was raised at a rate of $0.7^{\circ} \mathrm{C} / \mathrm{min}$. The data readings were taken every $60 \mathrm{~s}$. The melting temperatures $\left(T_{\mathrm{m}}\right)$ were determined from the inflection point of the melting curves by applying a first derivative calculation using the RotorGene 6000 application software.

Reverse transcriptase (RT) stop assay. DNA primer P18, 5'-TAATACGACTCACTATAG-3' (40 nM) was $5^{\prime}$-end labeled with $\left[\gamma^{-32}\right]$ ATP using T4 polynucleotide kinase and mixed with RNA template HT4, 5'-UCC AACUAUGUAUACUUAGGGUUAGGGUUAGGGUUAGGGACAUAUCGAUGAAAUUGCUAUAGUGAG UCGUAUUA-3' (40 nM) in $10 \mathrm{mM}$ Tris- $\mathrm{HCl}$ ( $\mathrm{pH} 8$ ) buffer containing $1.5 \mathrm{mM} \mathrm{MgCl}$, $35 \mathrm{mM} \mathrm{NaCl}$ and $5 \mathrm{mM}$ $\mathrm{KCl}$. The mixture was denatured by heating to $85^{\circ} \mathrm{C}$ and allowed to cool down to room temperature. Metallohelices at various concentrations were added to the mixture $(10 \mu \mathrm{L}$ final volume $)$ and incubated at room temperature for $10 \mathrm{~min}$. The primer extension reactions were initiated by adding $0.25 \mathrm{mM}$ dNTPs, $10 \mathrm{mM}$ dithiothreitol (DTT) and 10 units of ProtoScript II RT (recombinant M-MuLV reverse transcriptase with reduced RNase $\mathrm{H}$ activity and increased thermostability). After incubation at $42^{\circ} \mathrm{C}$ for $60 \mathrm{~min}$, the reactions were stopped by adding an equal volume of $2 \times$ concentrated formamide loading buffer and the products were separated on a $12 \%$ PAA sequencing gel.

RNase A digestion. 22_TERRA (the same oligoribonucleotide as for FID measurements) was $5^{\prime}$-end labeled using T4 polynucleotide kinase and $\left[\gamma^{-32} \mathrm{P}\right]$ ATP and annealed by heating at $85^{\circ} \mathrm{C}$ for $3 \mathrm{~min}$, followed by

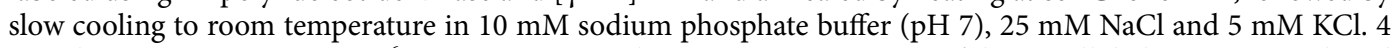
$\mu \mathrm{L}$ solutions containing $2 \times 10^{-6} \mathrm{M} 22$ _TERRA and various concentrations of the metallohelices were incubated 
for $10 \mathrm{~min}$ at room temperature. Cleavage was initiated by the addition of $1 \mu \mathrm{L}\left(\sim 6 \times 10^{-6}\right.$ units $)$ of RNase A. Samples were incubated for $12 \mathrm{~min}$ at room temperature before quenching with $5 \mu \mathrm{L}$ of $2 \times$ concentrated formamide loading buffer followed by heating at $90{ }^{\circ} \mathrm{C}$ for $3 \mathrm{~min}$. RNA cleavage products were resolved by PAA gel electrophoresis under denaturing conditions (24\%/8 M urea).

2-aminopurine (2Ap) fluorescence studies. The solutions of 2Ap-labeled oligoribonucleotides 22 TERRA_2Ap7，5'-AGGGUU2ApGGGUUAGGGUUAGGG-3', 22_TERRA_2Ap13, 5'-AGGGUUAGGGUU 2ApGGGUUAGGG-3', and 22_TERRA_2Ap19, 5'-AGGGUUAGGGUUAGGGUU2ApGGG-3' (40 $\mu$ M) were annealed in $10 \mathrm{mM}$ potassium phosphate ( $\mathrm{pH} 7.0$ ) and $10 \mathrm{mM} \mathrm{KCl}$ by heating to $85^{\circ} \mathrm{C}$ for $3 \mathrm{~min}$ followed by slow cooling to room temperature. Oligoribonucleotides $(2.5 \mu \mathrm{M})$ were mixed with $2.5 \mu \mathrm{M}$ or $5 \mu \mathrm{M}$ metallohelix in $10 \mathrm{mM}$ potassium phosphate $(\mathrm{pH} \mathrm{7.0)}$ and $10 \mathrm{mM} \mathrm{KCl}$ in a total volume of $80 \mu \mathrm{L}$ and placed in a quartz micro cuvette (volume of $50 \mu \mathrm{L}$ ). Samples were left undisturbed at room temperature for 5 min before taking measurement. Oligoribonucleotides $(2.5 \mu \mathrm{M})$ were titrated with metallohelices in a $0.5 \times 0.5 \mathrm{~cm}$ quartz cuvette in a total volume of $0.6 \mathrm{~mL}$ in $10 \mathrm{mM}$ potassium phosphate $(\mathrm{pH} \mathrm{7.0)}$ and $10 \mathrm{mM} \mathrm{KCl}$. Samples were thoroughly mixed by pipetting and kept undisturbed for $3 \mathrm{~min}$ at room temperature before data taking. Measurements were carried out using the Varian Cary Eclipse spectrofluorophotometer and the following parameters: the excitation and emission wavelengths were set to $310 \mathrm{~nm}$ and $365 \mathrm{~nm}$, respectively, widths of the excitation and emission slit were $10 \mathrm{~nm}$, and the averaging time was set to $3 \mathrm{~s}$.

Other physical methods. UV absorbance measurements were conducted on a Varian Cary 4000 UV/vis spectrophotometer. Gels containing radioactively labeled samples with $\left[\gamma^{-32} \mathrm{P}\right] \mathrm{ATP}$ were exposed to a phosphor imaging plate and scanned with a GE Healthcare FLA 7000 laser scanner.

Received: 20 May 2020; Accepted: 7 August 2020

Published online: 03 September 2020

\section{References}

1. Burge, S., Parkinson, G. N., Hazel, P., Todd, A. K. \& Neidle, S. Quadruplex DNA: Sequence, topology and structure. Nucleic Acids. Res. 34, 5402-5415 (2006).

2. Huppert, J. L. Four-stranded nucleic acids: Structure, function and targeting of G-quadruplexes. Chem. Soc. Rev. 37, 1375-1384 (2008).

3. Havrila, M. et al. Structural dynamics of propeller loop: Towards folding of RNA G-quadruplex. Nucleic Acids Res. 46, 8754-8771 (2018).

4. Xiao, C. D., Shibata, T., Yamamoto, Y. \& Xu, Y. An intramolecular antiparallel G-quadruplex formed by human telomere RNA. Chem. Commun. 54, 3944-3946 (2018).

5. Zaccaria, F. \& Fonseca Guerra, C. RNA versus DNA G-quadruplex: The origin of increased stability. Chem. Eur. J. 24, 16315-16322 (2018).

6. Beaudoin, J.-D. \& Perreault, J.-P. 5'-UTR G-quadruplex structures acting as translational repressors. Nucleic Acids. Res. 38, 7022$7036(2010)$.

7. Fay, M. M., Lyons, S. M. \& Ivanov, P. RNA G-quadruplexes in biology: Principles and molecular mechanisms. J. Mol. Biol. 429, 2127-2147 (2017).

8. Biffi, G., Di Antonio, M., Tannahill, D. \& Balasubramanian, S. Visualization and selective chemical targeting of RNA G-quadruplex structures in the cytoplasm of human cells. Nat. Chem. 6, 75-80 (2014).

9. Kwok, C. K. \& Balasubramanian, S. Targeted detection of G-quadruplexes in cellular RNAs. Angew. Chem. Int. Ed. 54, 6751-6754 (2015).

10. Kwok, C. K., Marsico, G., Sahakyan, A. B., Chambers, V. S. \& Balasubramanian, S. rG4-seq reveals widespread formation of G-quadruplex structures in the human transcriptome. Nat. Methods 13, 841 (2016).

11. Lipps, H. J. \& Rhodes, D. G-quadruplex structures: In vivo evidence and function. Trends Cell Biol. 19, 414-422 (2009).

12. Biffi, G., Tannahill, D., McCafferty, J. \& Balasubramanian, S. Quantitative visualization of DNA G-quadruplex structures in human cells. Nat. Chem. 5, $182(2013)$.

13. Bugaut, A. \& Balasubramanian, S. 5'-UTR RNA G-quadruplexes: Translation regulation and targeting. Nucleic Acids Res. 40, 4727-4741 (2012).

14. Jodoin, R., Carrier, J. C., Rivard, N., Bisaillon, M. \& Perreault, J.-P. G-quadruplex located in the 5'UTR of the BAG-1 mRNA affects both its cap-dependent and cap-independent translation through global secondary structure maintenance. Nucleic Acids Res. 47, 10247-10266 (2019).

15. Agarwala, P., Pandey, S. \& Maiti, S. The tale of RNA G-quadruplex. Org. Biomol. Chem. 13, 5570-5585 (2015).

16. Pandey, S., Agarwala, P., Jayaraj, G. G., Gargallo, R. \& Maiti, S. The RNA stem-loop to G-quadruplex equilibrium controls mature microRNA production inside the cell. Biochemistry 54, 7067-7078 (2015).

17. Perrone, R. et al. A dynamic G-quadruplex region regulates the HIV-1 long terminal repeat promoter. J. Med. Chem. 56, 6521-6530 (2013).

18. Krafćíková, P., Demkovičová, E. \& Víglaský, V. Ebola virus derived G-quadruplexes: Thiazole orange interaction. Biochim. Biophys. Acta 1861, 1321-1328 (2017).

19. Amrane, S. et al. Topology of a DNA G-quadruplex structure formed in the HIV-1 promoter: A potential target for anti-HIV drug development. J. Am. Chem. Soc. 136, 5249-5252 (2014).

20. Jaubert, C. et al. RNA synthesis is modulated by G-quadruplex formation in Hepatitis C virus negative RNA strand. Sci. Rep. 8, $8120(2018)$

21. Collie, G. W. \& Parkinson, G. N. The application of DNA and RNA G-quadruplexes to therapeutic medicines. Chem. Soc. Rev. 40, 5867-5892 (2011).

22. Ferino, A. et al. Photodynamic therapy for ras-driven cancers: Targeting G-quadruplex RNA structures with bifunctional alkylmodified porphyrins. J. Med. Chem. 63, 1245-1260 (2020).

23. Bugaut, A., Rodriguez, R., Kumari, S., Hsu, S.-T.D. \& Balasubramanian, S. Small molecule-mediated inhibition of translation by targeting a native RNA G-quadruplex. Org. Biomol. Chem. 8, 2771-2776 (2010). 
24. Collie, G., Reszka, A.P., Haider, S.M., Gabelica, V., Parkinson, G.N. \& Neidle, S. Selectivity in small molecule binding to human telomeric RNA and DNA quadruplexes. Chem. Commun. 14, 7482-7484 (2009).

25. Halder, K., Largy, E., Benzler, M., Teulade-Fichou, M.-P. \& Hartig, J. S. Efficient suppression of gene expression by targeting 5'-UTR-based RNA quadruplexes with bisquinolinium compounds. ChemBioChem 12, 1663-1668 (2011).

26. Wheelhouse, R. T., Sun, D., Han, H., Han, F. X. \& Hurley, L. H. Cationic porphyrins as telomerase inhibitors: The interaction of tetra-(N-methyl-4-pyridyl)porphine with quadruplex DNA. J. Am. Chem. Soc. 120, 3261-3262 (1998).

27. Izbicka, E. et al. Effects of cationic porphyrins as G-quadruplex interactive agents in human tumor cells. Cancer Res. 59, 639-644 (1999).

28. Ruan, T. L. et al. Lowering the overall charge on TMPyP4 improves its selectivity for G-quadruplex DNA. Biochimie 132, 121-130 (2017).

29. Morris, M. J., Wingate, K. L., Silwal, J., Leeper, T. C. \& Basu, S. The porphyrin TmPyP4 unfolds the extremely stable G-quadruplex in MT3-MMP mRNA and alleviates its repressive effect to enhance translation in eukaryotic cells. Nucleic Acids Res. 40, 4137-4145 (2012).

30. Zamiri, B., Reddy, K., Macgregor, R. B. \& Pearson, C. E. TMPyP4 porphyrin distorts RNA G-quadruplex structures of the diseaseassociated r(GGGGCC)n repeat of the C9orf72 gene and blocks interaction of RNA-binding proteins. J. Biol. Chem. 289, 4653-4659 (2014).

31. Vo, T. et al. Substituted naphthalenediimide compounds bind selectively to two human quadruplex structures with parallel topology. ACS Med. Chem. Lett. 11, 991-999 (2020).

32. Wang, J. S., Chen, Y., Ren, J. S., Zhao, C. Q. \& Qu, X. G. G-Quadruplex binding enantiomers show chiral selective interactions with human telomere. Nucleic Acids Res. 42, 3792-3802 (2014).

33. Yu, H., Wang, X., Fu, M., Ren, J. \& Qu, X. Chiral metallo-supramolecular complexes selectively recognize human telomeric G-quadruplex DNA. Nucleic Acids Res. 36, 5695-5703 (2008).

34. Zhao, A. et al. Chiral metallohelices enantioselectively target hybrid human telomeric G-quadruplex DNA. Nucleic Acids Res. 45, 5026-5035 (2017).

35. Howson, S. E. et al. Optically pure, water-stable metallo-helical "flexicate" assemblies with antibiotic activity. Nat. Chem. 4, 31-36 (2012).

36. Simpson, D. H. et al. Metallohelices that kill Gram-negative pathogens using intracellular antimicrobial peptide pathways. Chem. Sci. 10, 9708-9720 (2019).

37. Hrabina, O. et al. Optically pure metallohelices that accumulate in cell nuclei, condense/aggregate DNA, and inhibit activities of DNA processing enzymes. Inorg. Chem. 59, 3304-3311 (2020).

38. Martadinata, H. \& Phan, A. T. Structure of propeller-type parallel-stranded RNA G-quadruplexes, formed by human telomeric RNA sequences in $\mathrm{K}^{+}$solution. J. Am. Chem. Soc. 131, 2570-2578 (2009).

39. Collie, G. W., Haider, S. M., Neidle, S. \& Parkinson, G. N. A crystallographic and modelling study of a human telomeric RNA (TERRA) quadruplex. Nucleic Acids Res. 38, 5569-5580 (2010).

40. Xu, Y., Kaminaga, K. \& Komiyama, M. G-quadruplex formation by human telomeric repeats-containing $\mathrm{RNA}$ in $\mathrm{Na}^{+}$solution. J. Am. Chem. Soc. 130, 11179-11184 (2008).

41. Monchaud, D., Allain, C. \& Teulade-Fichou, M. P. Development of a fluorescent intercalator displacement assay (G4-FID) for establishing quadruplex-DNA affinity and selectivity of putative ligands. Bioorg. Med. Chem. Lett. 16, 4842-4845 (2006).

42. Rahman, K. M. et al. The prenylated dioxopiperazine alkaloid Cristatin A has selective telomeric DNA G-quadruplex stabilising properties. Chem. Commun. 48, 8760-8762 (2012).

43. Ang, D. L. et al. Quadruplex DNA-stabilising dinuclear platinum(II) terpyridine complexes with flexible linkers. Chem. Eur. J. 22, $2317-2325$ (2016).

44. Han, H. Y., Hurley, L. H. \& Salazar, M. A DNA polymerase stop assay for G-quadruplex-interactive compounds. Nucleic Acids Res. 27, 537-542 (1999).

45. Sielaff, A., Mackay, H., Brown, T. \& Lee, M. 2-aminopurine/cytosine base pair containing oligonucleotides: Fluorescence spectroscopy studies on DNA-polyamide binding. Biochem. Biophys. Res. Commun. 369, 630-634 (2008).

46. Bradrick, T. D. \& Marino, J. P. Ligand-induced changes in 2-aminopurine fluorescence as a probe for small molecule binding to HIV-1 TAR RNA. RNA 10, 1459-1468 (2004).

47. Holz, B., Weinhold, E., Klimasauskas, S. \& Serva, S. 2-Aminopurine as a fluorescent probe for DNA base flipping by methyltransferases. Nucleic Acids Res. 26, 1076-1083 (1998).

48. Rachofsky, E. L., Osman, R. \& Ross, J. B. A. Probing structure and dynamics of DNA with 2-aminopurine: Effects of local environment on fluorescence. Biochemistry 40, 946-956 (2001).

\section{Acknowledgements}

We acknowledge that Daniel H. Simpson from the Department of Chemistry, University of Warwick, Coventry, UK provided the metallohelices used in this study. This work was supported by the Czech Science Foundation [grant number 20-00735S].

\section{Author contributions}

J.M. was responsible for experimental design, data analysis, and wrote the manuscript. P.S. revised the manuscript. V.B. wrote and edited the manuscript. All authors reviewed the manuscript.

\section{Competing interests}

The authors declare no competing interests.

\section{Additional information}

Supplementary information is available for this paper at https://doi.org/10.1038/s41598-020-71429-5.

Correspondence and requests for materials should be addressed to V.B.

Reprints and permissions information is available at www.nature.com/reprints.

Publisher's note Springer Nature remains neutral with regard to jurisdictional claims in published maps and institutional affiliations. 
(c) (i) Open Access This article is licensed under a Creative Commons Attribution 4.0 International cc) License, which permits use, sharing, adaptation, distribution and reproduction in any medium or format, as long as you give appropriate credit to the original author(s) and the source, provide a link to the Creative Commons licence, and indicate if changes were made. The images or other third party material in this article are included in the article's Creative Commons licence, unless indicated otherwise in a credit line to the material. If material is not included in the article's Creative Commons licence and your intended use is not permitted by statutory regulation or exceeds the permitted use, you will need to obtain permission directly from the copyright holder. To view a copy of this licence, visit http://creativecommons.org/licenses/by/4.0/.

(C) The Author(s) 2020 